\title{
Selected papers from the 2019 Denver X-ray conference
}

This issue of Powder Diffraction (PDJ) contains six specially selected Technical Articles from the 2019 Denver X-ray Conference. These exceptional papers were selected by Tom Blanton, Lora Brehm, and Martina Schmeling, who subsequently served as guest Editors for PDJ.

Each of the six papers was presented in the 68th Denver X-Ray Conference held August 5-9, 2019, at the Westin Lombard Yorktown Center, Lombard, IL, USA (near Chicago). This meeting was held jointly with the 25th International Congress on X-ray Optics and Microanalysis. Selected papers from the ICXOM Congress will appear in a subsequent issue of Powder Diffraction. These six papers are an exceptional mix of state-of-the-art research and outstanding application of state-of-the-art XRD methods that should be of general interest to readers of Powder Diffraction.

The first paper is "Total Pattern Analyses for NonCrystalline Materials" by Timothy Fawcett et al., International Center for Diffraction Data. This paper describes a multi-year effort to collect and then add reference patterns of non-crystalline and amorphous materials to the Powder Diffraction File and to expand the tools included in the ICDD's PDF4+ computerized database and analysis tools to enable users of the PDF4+ database to conduct similar analyses. This paper describes the major advance by ICDD in both how to collect and record reference data and the development of computer tools for multi-phase ID analyses when non-crystalline/amorphous materials are present in the sample.

The second paper is "Use of a Be-Dome Holder for Texture and Strain Characterization of Li Metal Thin Films via the $\sin ^{2}(\psi)$ Methodology" by Mark Rodriguez et al. of Sandia National Laboratories. This paper provides a novel study of the methods for the characterization of deposition of thin Li metal, particularly as used for Li-ion batteries. The paper details sample preparation, alignment, and analysis of the air-sensitive Li films and shows that in-plane stresses change with applied pressure.

The third paper is "How to Use and How Not to Use Certified Reference Materials in Industrial Chemical Analysis" by John R. Sieber of the National Institutes for Standards and Technology. This paper discusses the need for and understanding the role for certified reference materials, particularly for accreditation and traceability to the International System of Units (SI). The concepts and guidance presented apply to chemical metrology and fundamental analytical chemistry with several examples from XRF.

The fourth paper is "Bi-Cross Validation of Spectral Clustering Hyperparameters" by S. Zohar and C. H. Yoon of the SLAC National Accelerator Laboratory. This X-ray source is sufficiently different from laboratory and conventional synchrotron sources that optimizing data analysis requires new methodologies to handle and help users interpret the terabyte scale X-ray scattering data obtained from the Stanford Linac Coherent Light Source (SLAC). While these methods may seem complex, as coherent light sources become more widely used, I anticipate we all will become interested in the use of such methodologies and X-ray sources in the future.

The fifth paper is "A Method for Mapping SubmicronScale Crystallographic Order/Disorder Applied to Human Tooth Enamel" by Robert Free et al. This paper discusses a method to characterize the population-level statistics of crystallographic features such as lattice parameter, crystallize size, and orientation distribution as a function of location. This paper describes a 2D diffraction methodology using $\sim 500 \mathrm{~nm}$ wide beam of monochromatic radiation in conjunction with a sample section of only $1 \mu \mathrm{m}$ in thickness. This "diffraction volume" involves several hundred crystallites and enables the extraction of statistics on crystallographic features such as lattice parameter, crystallite size, and orientation distributions as well as mapping order/disorder for correlation with the irradiated volume location within the enamel microstructure.

The sixth paper is " $\mathrm{X}$-Ray Diffraction as a Major Tool for the Analysis of $\mathrm{PM}_{2.5}$ and $\mathrm{PM}_{10}$ Aerosols" by Hamdan and Alawadhi, American University of Sharjah, United Arab Emirates. They showed that XRD methods can successfully be used to identify crystalline phases present, including secondary pollutants, in particulate matter filters collected from traffic sites. XRD data along with XRF, SEM, and matrix factorization modeling were combined to confirm that major phases identified (Mascagnite, Koktaite, and Gypsum) mostly originated from natural sources.

Following the six Technical Articles from 2019 DXC are three New Diffraction Data Articles and one Rapid Communications Article that provide very high-quality powder diffraction reference data. Three of these report data for important pharmaceutical compounds.

This issue of PDJ closes with an International Report on the 2019 Materials Science \& Technology Conference \& Exhibition (MS\&T19) and the Calendars of Forthcoming Meetings and of Upcoming Short Courses and Workshops.

Camden Hubbard

Editor-in-Chief, Powder Diffraction E-mail: camden.hubbard@me.com 